\title{
Lexicalized Stochastic Modeling of Constraint-Based Grammars using Log-Linear Measures and EM Training
}

\author{
Stefan Riezler \\ IMS, Universität Stuttgart \\ riezler@ims . uni-stuttgart.de \\ Jonas Kuhn \\ IMS, Universität Stuttgart \\ jonas@ims . uni-stuttgart.de
}

\begin{abstract}
We present a new approach to stochastic modeling of constraintbased grammars that is based on loglinear models and uses EM for estimation from unannotated data. The techniques are applied to an LFG grammar for German. Evaluation on an exact match task yields $86 \%$ precision for an ambiguity rate of 5.4 , and $90 \%$ precision on a subcat frame match for an ambiguity rate of 25 . Experimental comparison to training from a parsebank shows a $10 \%$ gain from EM training. Also, a new class-based grammar lexicalization is presented, showing a $10 \%$ gain over unlexicalized models.
\end{abstract}

\section{Introduction}

Stochastic parsing models capturing contextual constraints beyond the dependencies of probabilistic context-free grammars (PCFGs) are currently the subject of intensive research. An interesting feature common to most such models is the incorporation of contextual dependencies on individual head words into rulebased probability models. Such word-based lexicalizations of probability models are used successfully in the statistical parsing models of, e.g., Collins (1997), Charniak (1997), or Ratnaparkhi (1997). However, it is still an open question which kind of lexicalization, e.g., statistics on individual words or statistics based upon word classes, is the best choice. Secondly, these approaches have in common the fact that the probability models

\section{Detlef Prescher}

IMS, Universität Stuttgart

prescher@ims . uni-stuttgart.de

\section{Mark Johnson}

Cog. \& Ling. Sciences, Brown University

Mark_Johnson@brown .edu

are trained on treebanks, i.e., corpora of manually disambiguated sentences, and not from corpora of unannotated sentences. In all of the cited approaches, the Penn Wall Street Journal Treebank (Marcus et al., 1993) is used, the availability of which obviates the standard effort required for treebank training-handannotating large corpora of specific domains of specific languages with specific parse types. Moreover, common wisdom is that training from unannotated data via the expectationmaximization (EM) algorithm (Dempster et al., 1977) yields poor results unless at least partial annotation is applied. Experimental results confirming this wisdom have been presented, e.g., by Elworthy (1994) and Pereira and Schabes (1992) for EM training of Hidden Markov Models and PCFGs.

In this paper, we present a new lexicalized stochastic model for constraint-based grammars that employs a combination of headword frequencies and EM-based clustering for grammar lexicalization. Furthermore, we make crucial use of EM for estimating the parameters of the stochastic grammar from unannotated data. Our usage of EM was initiated by the current lack of large unificationbased treebanks for German. However, our experimental results also show an exception to the common wisdom of the insufficiency of EM for highly accurate statistical modeling.

Our approach to lexicalized stochastic modeling is based on the parametric family of loglinear probability models, which is used to define a probability distribution on the parses of a Lexical-Functional Grammar (LFG) for German. In previous work on log-linear models for LFG by Johnson et al. (1999), pseudo- 
likelihood estimation from annotated corpora has been introduced and experimented with on a small scale. However, to our knowledge, to date no large LFG annotated corpora of unrestricted German text are available. Fortunately, algorithms exist for statistical inference of log-linear models from unannotated data (Riezler, 1999). We apply this algorithm to estimate log-linear LFG models from large corpora of newspaper text. In our largest experiment, we used 250,000 parses which were produced by parsing 36,000 newspaper sentences with the German LFG. Experimental evaluation of our models on an exact-match task (i.e. percentage of exact match of most probable parse with correct parse) on 550 manually examined examples with on average 5.4 analyses gave $86 \%$ precision. Another evaluation on a verb frame recognition task (i.e. percentage of agreement between subcategorization frames of main verb of most probable parse and correct parse) gave $90 \%$ precision on 375 manually disambiguated examples with an average ambiguity of 25. Clearly, a direct comparison of these results to stateof-the-art statistical parsers cannot be made because of different training and test data and other evaluation measures. However, we would like to draw the following conclusions from our experiments:

- The problem of chaotic convergence behaviour of EM estimation can be solved for log-linear models.

- EM does help constraint-based grammars, e.g. using about 10 times more sentences and about 100 times more parses for EM training than for training from an automatically constructed parsebank can improve precision by about $10 \%$.

- Class-based lexicalization can yield a gain in precision of about $10 \%$.

In the rest of this paper we introduce incomplete-data estimation for log-linear models (Sec. 2), and present the actual design of our models (Sec. 3) and report our experimental results (Sec. 4).

\section{Incomplete-Data Estimation for Log-Linear Models}

\section{$2.1 \quad$ Log-Linear Models}

A log-linear distribution $p_{\lambda}(x)$ on the set of analyses $\mathcal{X}$ of a constraint-based grammar can be defined as follows:

$$
p_{\lambda}(x)=Z_{\lambda}^{-1} e^{\lambda \cdot \nu(x)} p_{0}(x)
$$

where $Z_{\lambda}=\sum_{x \in \mathcal{X}} e^{\lambda \cdot \nu(x)} p_{0}(x)$ is a normalizing constant, $\lambda=\left(\lambda_{1}, \ldots, \lambda_{n}\right) \in \mathbb{R}^{n}$ is a vector of log-parameters, $\nu=\left(\nu_{1}, \ldots, \nu_{n}\right)$ is a vector of property-functions $\nu_{i}: \mathcal{X} \rightarrow \mathbb{R}$ for $i=1, \ldots, n, \lambda \cdot \nu(x)$ is the vector dot product $\sum_{i=1}^{n} \lambda_{i} \nu_{i}(x)$, and $p_{0}$ is a fixed reference distribution.

The task of probabilistic modeling with loglinear distributions is to build salient properties of the data as property-functions $\nu_{i}$ into the probability model. For a given vector $\nu$ of property-functions, the task of statistical inference is to tune the parameters $\lambda$ to best reflect the empirical distribution of the training data.

\subsection{Incomplete-Data Estimation}

Standard numerical methods for statistical inference of log-linear models from fully annotated data-so-called complete data-are the iterative scaling methods of Darroch and Ratcliff (1972) and Della Pietra et al. (1997). For data consisting of unannotated sentences-so-called incomplete data - the iterative method of the EM algorithm (Dempster et al., 1977) has to be employed. However, since even complete-data estimation for log-linear models requires iterative methods, an application of EM to log-linear models results in an algorithm which is expensive since it is doubly-iterative. A singly-iterative algorithm interleaving EM and iterative scaling into a mathematically well-defined estimation method for log-linear models from incomplete data is the IM algorithm of Riezler (1999). Applying this algorithm to stochastic constraint-based grammars, we assume the following to be given: A training sample of unannotated sentences $y$ from a set $\mathcal{Y}$, observed with empirical 


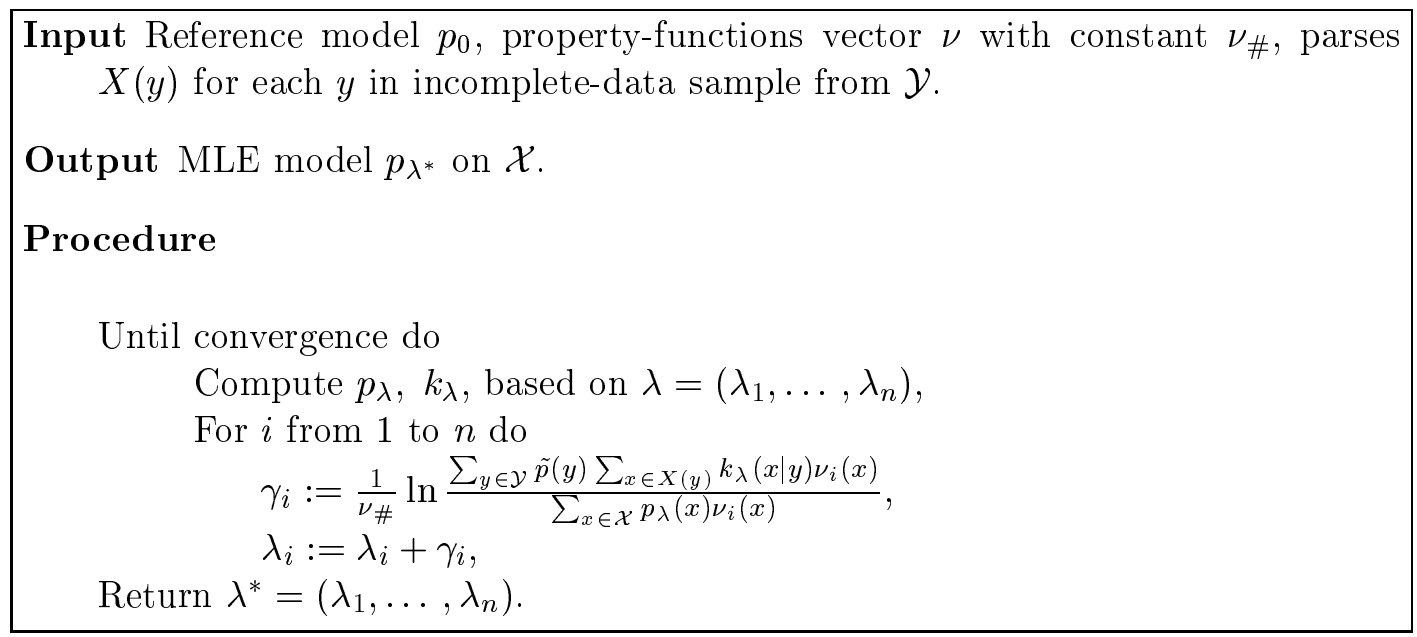

Figure 1: Closed-form version of IM algorithm

probability $\tilde{p}(y)$, a constraint-based grammar yielding a set $X(y)$ of parses for each sentence $y$, and a log-linear model $p_{\lambda}(\cdot)$ on the parses $\mathcal{X}=\sum_{y \in \mathcal{Y} \mid \tilde{p}(y)>0} X(y)$ for the sentences in the training corpus, with known values of property-functions $\nu$ and unknown values of $\lambda$. The aim of incomplete-data maximum likelihood estimation (MLE) is to find a value $\lambda^{*}$ that maximizes the incomplete-data loglikelihood $L=\sum_{y \in \mathcal{Y}} \tilde{p}(y) \ln \sum_{x \in X(y)} p_{\lambda}(x)$, i.e.,

$$
\lambda^{*}=\underset{\lambda \in \mathbb{R}^{n}}{\arg \max } L(\lambda) .
$$

Closed-form parameter-updates for this problem can be computed by the algorithm of Fig. 1 , where $\nu_{\#}(x)=\sum_{i=1}^{n} \nu_{i}(x)$, and $k_{\lambda}(x \mid y)=$ $p_{\lambda}(x) / \sum_{x \in X(y)} p_{\lambda}(x)$ is the conditional probability of a parse $x$ given the sentence $y$ and the current parameter value $\lambda$.

The constancy requirement on $\nu_{\#}$ can be enforced by adding a "correction" propertyfunction $\nu_{l}$ :

Choose $K=\max _{x \in \mathcal{X}} \nu_{\#}(x)$ and $\nu_{l}(x)=K-\nu_{\#}(x)$ for all $x \in \mathcal{X}$.

Then $\sum_{i=1}^{l} \nu_{i}(x)=K$ for all $x \in \mathcal{X}$.

Note that because of the restriction of $\mathcal{X}$ to the parses obtainable by a grammar from the training corpus, we have a log-linear probability measure only on those parses and not on all possible parses of the grammar. We shall therefore speak of mere log-linear measures in our application of disambiguation.

\subsection{Searching for Order in Chaos}

For incomplete-data estimation, a sequence of likelihood values is guaranteed to converge to a critical point of the likelihood function $L$. This is shown for the IM algorithm in Riezler (1999). The process of finding likelihood maxima is chaotic in that the final likelihood value is extremely sensitive to the starting values of $\lambda$, i.e. limit points can be local maxima (or saddlepoints), which are not necessarily also global maxima. A way to search for order in this chaos is to search for starting values which are hopefully attracted by the global maximum of $L$. This problem can best be explained in terms of the minimum divergence paradigm (Kullback, 1959), which is equivalent to the maximum likelihood paradigm by the following theorem. Let $p[f]=\sum_{x \in \mathcal{X}} p(x) f(x)$ be the expectation of a function $f$ with respect to a distribution $p$ :

The probability distribution $p^{*}$ that minimizes the divergence $D\left(p \| p_{0}\right)$ to a reference model $p_{0}$ subject to the constraints $p\left[\nu_{i}\right]=q\left[\nu_{i}\right], i=1, \ldots, n$ is the model in the parametric family of log-linear distributions $p_{\lambda}$ that maximizes the likelihood $L(\lambda)=$ $q\left[\ln p_{\lambda}\right]$ of the training data ${ }^{1}$.

\footnotetext{
${ }^{1}$ If the training sample consists of complete data
} 
Reasonable starting values for minimum divergence estimation is to set $\lambda_{i}=0$ for $i=1, \ldots, n$. This yields a distribution which minimizes the divergence to $p_{0}$, over the set of models $p$ to which the constraints $p\left[\nu_{i}\right]=q\left[\nu_{i}\right], i=1, \ldots, n$ have yet to be applied. Clearly, this argument applies to both complete-data and incomplete-data estimation. Note that for a uniformly distributed reference model $p_{0}$, the minimum divergence model is a maximum entropy model (Jaynes, 1957). In Sec. 4, we will demonstrate that a uniform initialization of the IM algorithm shows a significant improvement in likelihood maximization as well as in linguistic performance when compared to standard random initialization.

\section{Property Design and Lexicalization}

\subsection{Basic Configurational Properties}

The basic 190 properties employed in our models are similar to the properties of Johnson et al. (1999) which incorporate general linguistic principles into a log-linear model. They refer to both the c(onstituent)structure and the $f($ eature)-structure of the LFG parses. Examples are properties for

- c-structure nodes, corresponding to standard production properties,

- c-structure subtrees, indicating argument versus adjunct attachment,

- f-structure attributes, corresponding to grammatical functions used in LFG,

- atomic attribute-value pairs in fstructures,

- complexity of the phrase being attached to, thus indicating both high and low attachment,

- non-right-branching behavior of nonterminal nodes,

- non-parallelism of coordinations.

$x \in \mathcal{X}$, the expectation $q[\cdot]$ corresponds to the empirical expectation $\tilde{p}[\cdot]$. If we observe incomplete data $y \in \mathcal{Y}$, the expectation $q[\cdot]$ is replaced by the conditional expectation $\tilde{p}\left[k_{\lambda^{\prime}}[\cdot]\right]$ given the observed data $y$ and the current parameter value $\lambda^{\prime}$.

\subsection{Class-Based Lexicalization}

Our approach to grammar lexicalization is class-based in the sense that we use classbased estimated frequencies $f_{c}(v, n)$ of headverbs $v$ and argument head-nouns $n$ instead of pure frequency statistics or classbased probabilities of head word dependencies. Class-based estimated frequencies are introduced in Prescher et al. (2000) as the frequency $f(v, n)$ of a $(v, n)$-pair in the training corpus, weighted by the best estimate of the class-membership probability $p(c \mid v, n)$ of an EM-based clustering model on $(v, n)$-pairs, i.e., $f_{c}(v, n)=\max _{c \in C} p(c \mid v, n)(f(v, n)+1)$. As is shown in Prescher et al. (2000) in an evaluation on lexical ambiguity resolution, a gain of about $7 \%$ can be obtained by using the class-based estimated frequency $f_{c}(v, n)$ as disambiguation criterion instead of classbased probabilities $p(n \mid v)$. In order to make the most direct use possible of this fact, we incorporated the decisions of the disambiguator directly into 45 additional properties for the grammatical relations of the subject, direct object, indirect object, infinitival object, oblique and adjunctival dative and accusative preposition, for active and passive forms of the first three verbs in each parse. Let $v_{r}(x)$ be the verbal head of grammatical relation $r$ in parse $x$, and $n_{r}(x)$ the nominal head of grammatical relation $r$ in $x$. Then a lexicalized property $\nu_{r}$ for grammatical relation $r$ is defined as

$\nu_{r}(x)=\left\{\begin{array}{cc}1 & \text { if } f_{c}\left(v_{r}(x), n_{r}(x)\right) \geq \\ & f_{c}\left(v_{r}\left(x^{\prime}\right), n_{r}\left(x^{\prime}\right)\right) \forall x^{\prime} \in X(y) \\ 0 & \text { otherwise. }\end{array}\right.$

The property-function $\nu_{r}$ thus predisambiguates the parses $x \in X(y)$ of a sentence $y$ according to $f_{c}(v, n)$, and stores the best parse directly instead of taking the actual estimated frequencies as its value. In Sec. 4, we will see that an incorporation of this pre-disambiguation routine into the models improves performance in disambiguation by about $10 \%$. 


\begin{tabular}{|c|l|c|c|}
\hline $\begin{array}{c}\text { exact match } \\
\text { evaluation }\end{array}$ & $\begin{array}{c}\text { basic } \\
\text { model }\end{array}$ & $\begin{array}{c}\text { lexicalized } \\
\text { model }\end{array}$ & $\begin{array}{c}\text { selected } \\
+ \text { lexicalized } \\
\text { model }\end{array}$ \\
\hline $\begin{array}{c}\text { complete-data } \\
\text { estimation }\end{array}$ & P: 68 & P: 73.9 & P: 74.3 \\
\hline incomplete-data & P: 73.6 & E: 71.6 & E: 71.8 \\
estimation & E: 65.4 & E: 86 & P: 86.1 \\
\hline
\end{tabular}

Figure 2: Evaluation on exact match task for 550 examples with average ambiguity 5.4

\begin{tabular}{|c|c|c|c|}
\hline $\begin{array}{c}\text { frame match } \\
\text { evaluation }\end{array}$ & $\begin{array}{c}\text { basic } \\
\text { model }\end{array}$ & $\begin{array}{c}\text { lexicalized } \\
\text { model }\end{array}$ & $\begin{array}{c}\text { selected } \\
\text { lexicalized } \\
\text { model }\end{array}$ \\
\hline $\begin{array}{c}\text { complete-data } \\
\text { estimation }\end{array}$ & P: 80.6 & P: 82.7 & P: 83.4 \\
\hline E: 70.4 & E: 76.4 & E: 76 \\
estimation & P: 84.5 & P: 88.5 & P: 90 \\
E: 73.1 & E: 84.9 & E: 86.3 \\
\hline
\end{tabular}

Figure 3: Evaluation on frame match task for 375 examples with average ambiguity 25

\section{Experiments}

\subsection{Incomplete Data and Parsebanks}

In our experiments, we used an LFG grammar for German ${ }^{2}$ for parsing unrestricted text. Since training was faster than parsing, we parsed in advance and stored the resulting packed c/f-structures. The low ambiguity rate of the German LFG grammar allowed us to restrict the training data to sentences with at most 20 parses. The resulting training corpus of unannotated, incomplete data consists of approximately 36,000 sentences of online available German newspaper text, comprising approximately 250,000 parses.

In order to compare the contribution of unambiguous and ambiguous sentences to the estimation results, we extracted a subcorpus of 4,000 sentences, for which the LFG grammar produced a unique parse, from the full train-

\footnotetext{
${ }^{2}$ The German LFG grammar is being implemented in the Xerox Linguistic Environment (XLE, see Maxwell and Kaplan (1996)) as part of the Parallel Grammar (ParGram) project at the IMS Stuttgart. The coverage of the grammar is about $50 \%$ for unrestricted newspaper text. For the experiments reported here, the effective coverage was lower, since the corpus preprocessing we applied was minimal. Note that for the disambiguation task we were interested in, the overall grammar coverage was of subordinate relevance.
}

ing corpus. The average sentence length of 7.5 for this automatically constructed parsebank is only slightly smaller than that of 10.5 for the full set of 36,000 training sentences and 250,000 parses. Thus, we conjecture that the parsebank includes a representative variety of linguistic phenomena. Estimation from this automatically disambiguated parsebank enjoys the same complete-data estimation properties ${ }^{3}$ as training from manually disambiguated treebanks. This makes a comparison of complete-data estimation from this parsebank to incomplete-data estimation from the full set of training data interesting.

\subsection{Test Data and Evaluation Tasks}

To evaluate our models, we constructed two different test corpora. We first parsed with the LFG grammar 550 sentences which are used for illustrative purposes in the foreign language learner's grammar of Helbig and Buscha (1996). In a next step, the correct parse was indicated by a human disambiguator, according to the reading intended in Helbig and Buscha (1996). Thus a precise

\footnotetext{
${ }^{3}$ For example, convergence to the global maximum of the complete-data log-likelihood function is guaranteed, which is a good condition for highly precise statistical disambiguation.
} 
indication of correct $\mathrm{c} / \mathrm{f}$-structure pairs was possible. However, the average ambiguity of this corpus is only 5.4 parses per sentence, for sentences with on average 7.5 words. In order to evaluate on sentences with higher ambiguity rate, we manually disambiguated further 375 sentences of LFG-parsed newspaper text. The sentences of this corpus have on average 25 parses and 11.2 words.

We tested our models on two evaluation tasks. The statistical disambiguator was tested on an "exact match" task, where exact correspondence of the full c/f-structure pair of the hand-annotated correct parse and the most probable parse is checked. Another evaluation was done on a "frame match" task, where exact correspondence only of the subcategorization frame of the main verb of the most probable parse and the correct parse is checked. Clearly, the latter task involves a smaller effective ambiguity rate, and is thus to be interpreted as an evaluation of the combined system of highly-constrained symbolic parsing and statistical disambiguation.

Performance on these two evaluation tasks was assessed according to the following evaluation measures:

Precision $=\frac{\# \text { correct }}{\text { \#correct+\#incorrect }}$,
Effectiveness $=\frac{\text { \#correct }}{\# \text { correct+\#incorrect+\#don't know }}$. "Correct" and "incorrect" specifies a success/failure on the respective evaluation tasks; "don't know" cases are cases where the system is unable to make a decision, i.e. cases with more than one most probable parse.

\subsection{Experimental Results}

For each task and each test corpus, we calculated a random baseline by averaging over several models with randomly chosen parameter values. This baseline measures the disambiguation power of the pure symbolic parser. The results of an exact-match evaluation on the Helbig-Buscha corpus is shown in Fig. 2. The random baseline was around $33 \%$ for this case. The columns list different models according to their property-vectors. "Basic" models consist of 190 configurational properties as described in Sec. 3.1. "Lexical- ized" models are extended by 45 lexical predisambiguation properties as described in Sec. 3.2. "Selected + lexicalized" models result from a simple property selection procedure where a cutoff on the number of parses with non-negative value of the property-functions was set. Estimation of basic models from complete data gave $68 \%$ precision $(\mathrm{P})$, whereas training lexicalized and selected models from incomplete data gave $86.1 \%$ precision, which is an improvement of $18 \%$. Comparing lexicalized models in the estimation method shows that incomplete-data estimation gives an improvement of $12 \%$ precision over training from the parsebank. A comparison of models trained from incomplete data shows that lexicalization yields a gain of $13 \%$ in precision. Note also the gain in effectiveness (E) due to the pre-disambigution routine included in the lexicalized properties. The gain due to property selection both in precision and effectiveness is minimal. A similar pattern of performance arises in an exact match evaluation on the newspaper corpus with an ambiguity rate of 25 . The lexicalized and selected model trained from incomplete data achieved here $60.1 \%$ precision and $57.9 \%$ effectiveness, for a random baseline of around $17 \%$.

As shown in Fig. 3, the improvement in performance due to both lexicalization and EM training is smaller for the easier task of frame evaluation. Here the random baseline is $70 \%$ for frame evaluation on the newspaper corpus with an ambiguity rate of 25 . An overall gain of roughly $10 \%$ can be achieved by going from unlexicalized parsebank models (80.6\% precision) to lexicalized EM-trained models (90\% precision). Again, the contribution to this improvement is about the same for lexicalization and incomplete-data training. Applying the same evaluation to the Helbig-Buscha corpus shows $97.6 \%$ precision and $96.7 \%$ effectiveness for the lexicalized and selected incompletedata model, compared to around $80 \%$ for the random baseline.

Optimal iteration numbers were decided by repeated evaluation of the models at every fifth iteration. Fig. 4 shows the precision of lexicalized and selected models on the exact 


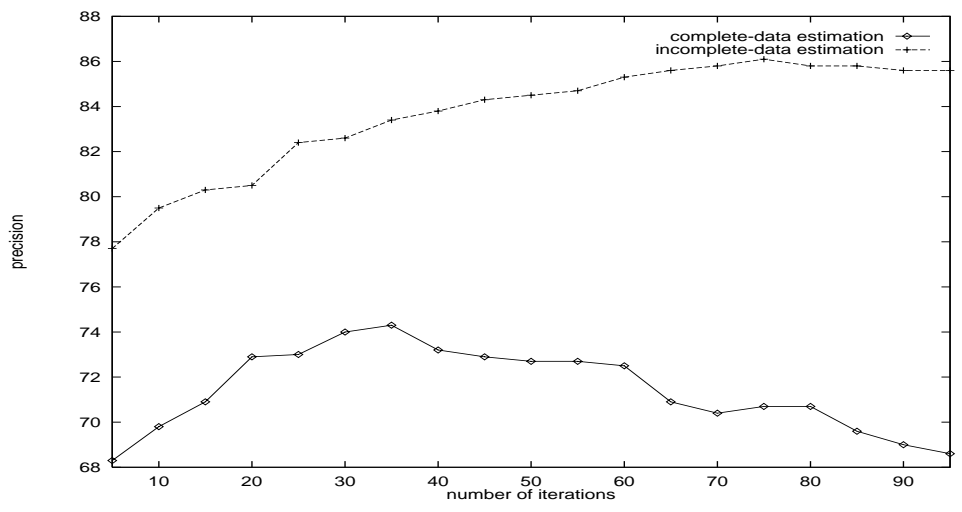

Figure 4: Precision on exact match task in number of training iterations

match task plotted against the number of iterations of the training algorithm. For parsebank training, the maximal precision value is obtained at 35 iterations. Iterating further shows a clear overtraining effect. For incomplete-data estimation more iterations are necessary to reach a maximal precision value. A comparison of models with random or uniform starting values shows an increase in precision of $10 \%$ to $40 \%$ for the latter. In terms of maximization of likelihood, this corresponds to the fact that uniform starting values immediately push the likelihood up to nearly its final value, whereas random starting values yield an initial likelihood which has to be increased by factors of 2 to 20 to an often lower final value.

\section{Discussion}

The most direct points of comparison of our method are the approaches of Johnson et al. (1999) and Johnson and Riezler (2000). In the first approach, log-linear models on LFG grammars using about 200 configurational properties were trained on treebanks of about 400 sentences by maximum pseudo-likelihood estimation. Precision was evaluated on an exact match task in a 10 -way cross validation paradigm for an ambiguity rate of 10 , and achieved $59 \%$ for the first approach. Johnson and Riezler (2000) achieved a gain of $1 \%$ over this result by including a classbased lexicalization. Our best models clearly outperform these results, both in terms of precision relative to ambiguity and in terms of relative gain due to lexicalization. A comparison of performance is more difficult for the lexicalized PCFG of Beil et al. (1999) which was trained by EM on 450,000 sentences of German newspaper text. There, a $70.4 \%$ precision is reported on a verb frame recognition task on 584 examples. However, the gain achieved by Beil et al. (1999) due to grammar lexicalizaton is only $2 \%$, compared to about $10 \%$ in our case. A comparison is difficult also for most other state-of-theart PCFG-based statistical parsers, since different training and test data, and most importantly, different evaluation criteria were used. A comparison of the performance gain due to grammar lexicalization shows that our results are on a par with that reported in Charniak (1997).

\section{Conclusion}

We have presented a new approach to stochastic modeling of constraint-based grammars. Our experimental results show that EM training can in fact be very helpful for accurate stochastic modeling in natural language processing. We conjecture that this result is due partly to the fact that the space of parses produced by a constraint-based grammar is only "mildly incomplete", i.e. the ambiguity rate can be kept relatively low. Another reason may be that EM is especially useful for log-linear models, where the search space in maximization can be kept under control. Furthermore, we have introduced a new class- 
based grammar lexicalization, which again uses EM training and incorporates a predisambiguation routine into log-linear models. An impressive gain in performance could also be demonstrated for this method. Clearly, a central task of future work is a further exploration of the relation between complete-data and incomplete-data estimation for larger, manually disambiguated treebanks. An interesting question is whether a systematic variation of training data size along the lines of the EM-experiments of Nigam et al. (2000) for text classification will show similar results, namely a systematic dependence of the relative gain due to EM training from the relative sizes of unannotated and annotated data. Furthermore, it is important to show that EMbased methods can be applied successfully also to other statistical parsing frameworks.

\section{Acknowledgements}

We thank Stefanie Dipper and Bettina Schrader for help with disambiguation of the test suites, and the anonymous ACL reviewers for helpful suggestions. This research was supported by the ParGram project and the project B7 of the SFB 340 of the DFG.

\section{References}

Franz Beil, Glenn Carroll, Detlef Prescher, Stefan Riezler, and Mats Rooth. 1999. Inside-outside estimation of a lexicalized PCFG for German. In Proceedings of the 37th ACL, College Park, MD.

Eugene Charniak. 1997. Statistical parsing with a context-free grammar and word statistics. In Proceedings of the 14th AAAI, Menlo Park, CA.

Michael Collins. 1997. Three generative, lexicalised models for statistical parsing. In Proceedings of the 35th ACL, Madrid.

J.N. Darroch and D. Ratcliff. 1972. Generalized iterative scaling for log-linear models. The Annals of Mathematical Statistics, 43(5):14701480 .

Stephen Della Pietra, Vincent Della Pietra, and John Lafferty. 1997. Inducing features of random fields. IEEE PAMI, 19(4):380-393.
A. P. Dempster, N. M. Laird, and D. B. Rubin. 1977. Maximum likelihood from incomplete data via the $E M$ algorithm. Journal of the Royal Statistical Society, 39(B):1-38.

David Elworthy. 1994. Does Baum-Welch reestimation help taggers? In Proceedings of the 4th ANLP, Stuttgart.

Gerhard Helbig and Joachim Buscha. 1996. Deutsche Grammatik. Ein Handbuch für den Ausländerunterricht. Langenscheidt, Leipzig.

Edwin T. Jaynes. 1957. Information theory and statistical mechanics. Physical Review, 106:620-630.

Mark Johnson and Stefan Riezler. 2000. Exploiting auxiliary distributions in stochastic unification-based grammars. In Proceedings of the 1st NAACL, Seattle, WA.

Mark Johnson, Stuart Geman, Stephen Canon, Zhiyi Chi, and Stefan Riezler. 1999. Estimators for stochastic "unification-based" grammars. In Proceedings of the 37th ACL, College Park, MD.

Solomon Kullback. 1959. Information Theory and Statistics. Wiley, New York.

Mitchell P. Marcus, Beatrice Santorini, and Mary Ann Marcinkiewicz. 1993. Building a large annotated corpus of english: The Penn treebank. Computational Linguistics, 19(2):313-330.

John Maxwell and R. Kaplan. 1996. Unificationbased parsers that automatically take advantage of context freeness. Unpublished manuscript, Xerox Palo Alto Research Center.

Kamal Nigam, Andrew McCallum, Sebastian Thrun, and Tom Mitchell. 2000. Text classification from labeled and unlabeled documents using EM. Machine Learning, 39(2/4):103-134.

Fernando Pereira and Yves Schabes. 1992. Insideoutside reestimation from partially bracketed corpora. In Proceedings of the 30th ACL, Newark, Delaware.

Detlef Prescher, Stefan Riezler, and Mats Rooth. 2000. Using a probabilistic class-based lexicon for lexical ambiguity resolution. In Proceedings of the 18th COLING, Saarbrücken.

Adwait Ratnaparkhi. 1997. A linear observed time statistical parser based on maximum entropy models. In Proceedings of EMNLP-2.

Stefan Riezler. 1999. Probabilistic Constraint Logic Programming Ph.D. thesis, Seminar für Sprachwissenschaft, Universität Tübingen. AIMS Report, 5(1), IMS, Universität Stuttgart. 